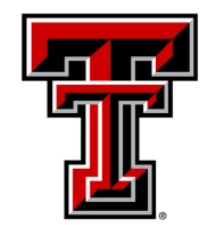

TEXAS TECH UNIVERSITY

Libraries"

\title{
THE EFFECTS OF TAI CHI ON MEASURES OF STRESS AND COPING STYLE
}

The Texas Tech community has made this publication openly available. Please share how this access benefits you. Your story matters to us.

\begin{tabular}{|l|l|}
\hline Citation & $\begin{array}{l}\text { Robert-McComb, J. J., Chyu, M. C., Tacón, A., \& Norman, R. (2015). } \\
\text { The effects of tai chi on measures of stress and coping style. Focus } \\
\text { on Alternative and Complementary Therapies, 20(2), 89-96. } \\
\text { https://doi.org/10.1111/fct.12179 }\end{array}$ \\
\hline Citable Link & $\underline{\text { http://hdl.handle.net/2346/73657 }}$ \\
\hline Terms of Use & $\underline{\text { CC-BY }}$ \\
\hline
\end{tabular}


ORIGINAL ARTICLE

Focus on Alternative and

Complementary Therapies

Volume 20(2) June 2015

C2015 Royal Pharmaceutical Society

DOI $10.1111 /$ fct.12177

ISSN 1465-3753

\title{
The effects of tai chi on measures of stress and coping style
}

\author{
Jacalyn J Robert-McComb, Ming Chyu, Anna Tacón, Reid Norman
}

\begin{abstract}
Background The biological response (heart-rate variability; HRV) to an acute psychological stressor, as well as cognitive changes in anxiety and coping style following tai chi (TC) in healthy male college students, has not been investigated in the literature.

Objectives The purpose of this study was to examine the effects of TC on: (1) HRV biomarkers of acute psychological stress, (2) state anxiety, and (3) coping style in healthy male college students.

Methods The study was an randomized clinical trial (RCT), in which the experimental group $(n=9)$ received TC (twice a week for 8 weeks) and the control (C) group $(n=11)$ did not receive treatment. Reactions to an acute psychological stressor were measured by the HRV's Standard Deviation of Normal to Normal Intervals (SDNN), and Spielberger's State-Trait Anxiety Inventory (STAI). Four 'trials' (baseline; stressor; recovery 1 and recovery 2) were conducted, with pre-tests/post-tests performed for both study groups. The Problem-Focused Styles of Coping (PFSOC) questionnaire was used to examine coping styles pre-test/post-test for both groups.

Results Significant differences were found between groups, including: (1) for group, test, and trial, the interaction of trial by group, and the interaction of test by trial for SDNN, $P<0.05$; (2) for test, trial, and the interaction of test by group for STAI $(P<0.05)$; and (3) for the interaction of group and test for the suppressive coping style $(P<0.05)$.

Conclusions Tai chi affects biological reactions to an acute psychological stressor, perceptions of anxiety to an acute stressor and coping styles in healthy male college students.
\end{abstract}

Keywords

Coping $\bullet$ complementary and alternative medicine $\bullet$ heart-rate variability $\bullet$ stress $\bullet$ tai chi

\section{Introduction}

The integrated action of adaptive biological and psychological responses promote successful coping, and physical activity is a positive way to improve health and reduce stress. Otherwise, ineffective coping with daily challenges can result in chronic disease if allostatic load is excessive. ' The concept of 'allostatic load,' proposed by McEwen and Stellar in 1993, refers to the biological wear and tear the body experiences due to repeated cycles of allostasis, which include the inefficient turning on and shutting off of such mechanisms in response to stress. $^{2}$ Allostasis means 'maintaining stability (or homeostasis) through change' or 
'remaining stable by being variable.' ${ }^{3}$ Physical activity, especially mind-body exercise, can be a way of coping with stressors in life and, if practised regularly, can result in an adaptive physiological response to maintain homeostasis when exposed to a psychological stressor.

Therefore, the aim of this study was to investigate the effect of a physical activity, tai chi (TC), on: (a) a biological marker (heart-rate variability; HRV) of acute psychological stress, (b) perceived anxiety or state anxiety when exposed to an acute psychological stressor, and (c) coping style. Adaptive changes to these variables pre-test/post-test TC were examined. Of special interest was the variable HRV.

Heart-rate variability is the term used to describe the 'oscillation in the interval between consecutive heartbeats as well as oscillations between consecutive instantaneous heart rates. ${ }^{5}$ Reduced HRV [beat-to-beat alterations in heart rate (HR)] is a sign of poor homeostatic control and represents the body's inability to maintain homeostasis through variability. ${ }^{4} \mathrm{~A}$ valid and reliable measurement of HRV is the standard deviation of the measure of time between two consecutive sinus heart beats or the variance in the inter-beat interval (IBI). It is customary to use the normal to normal $(\mathrm{NN})$ qualifier of IBI. This time domain variable of HRV is referred to as the Standard Deviation of Normal to Normal interval (SDNN); mathematically, it represents the square root of variance, with SDNN reflecting all the cyclic components responsible for variability in the period of recording. It is a common biological measure of the stress response, thus the adaptive changes in HRV following TC were of special interest to the researchers. ${ }^{5}$ Numerous studies have investigated the effect of TC on improvements in health in the elderly and other disease populations, but few studies have looked at the impact of TC on young male college students. ${ }^{6}$ Specifically, the biological response (via HRV) to an acute stressor and cognitive changes in anxiety and coping skills following TC has not been examined.

Therefore, the purpose of this study was threefold: (1) to examine the effects of TC on biomarkers of acute psychological stress using the HRV metric, SDNN; (2) to examine the effects of TC on state anxiety using STAI; ${ }^{7}$ and (3) to measure changes in coping style using the Problem-Focused Styles of Coping (PFSOC) questionnaire following TC. The questionnaire assesses three different coping styles or strategies: suppressive (PFSOC-SP), reflective (PFSOC-RF) and reactive (PFSOC-RC). ${ }^{8}$

It was hypothesised that: (1) SDNN would increase following intervention in the TC group but not the control group; (2) state anxiety would decrease following intervention in the TC group but not the control group; and (3) coping styles would change in the TC group but not in the control group following intervention.

\section{Methods \\ Study design}

The study was an randomized clinical trial (RCT). The experimental group received TC and the control group did not receive any treatment.

\section{Participants}

All participants signed the consent form as approved by the Texas Tech University Institutional Review Board, USA, before screening. The inclusion criteria were that all participants must: (a) be male college students aged between 18 and 45 years; (b) answer no to all of the questions on the Physical Activity Readiness Questionnaire (PAR-Q) ${ }^{9}$ (c) not have previously practised mind-body exercise or meditation; and (d) not be taking medications that would affect HR. 
Participants were normal, healthy male college students without any signs and symptoms of heart disease as measured by the PAR-Q. Subjects were randomly selected to participate in the control or TC group. Each participant's study number was placed in a hat. The first drawn study number was assigned to the TC group, the second drawn study number was control, and so on, until all study numbers were drawn.

\section{Pre-test/post-test procedures}

Participants were asked to refrain from all food, alcohol, caffeine, chewing gum, or the use of tobacco products $3 \mathrm{~h}$ before their scheduled appointments. They were also asked to avoid significant exertion or exercise on the day of the assessments. When participants arrived at the testing site, a checklist was completed to ensure these criteria were met.

Each testing session took approximately 2-2.5 hours. All testing was conducted between 1300 and $1700 \mathrm{~h}$ in a laboratory setting. Participants were asked to sit in a hospital patient recliner with the back in a vertical resting position. Pre-tests/post-tests were conducted on the same day and time for each individual participant.

Biofeedback Equipment, Model 1-330-C2+ (J \& J Engineering; Poulsbo, Washington, USA) was used to collect HRV data at 1024 samples per second for high resolution spectral displays and precision filtering. Participants completed a hard copy of the PFSOC questionnaire before any sensors were placed on their body. ${ }^{8}$ Sensors were placed on the participants' wrist and a respiration sensor around the chest. The timeline for both the pre-test and post-test was as follows: $20 \mathrm{~min}$ for baseline data; $10 \mathrm{~min}$ of rest; $20 \mathrm{~min}$ for the psychological stressor; $20 \mathrm{~min}$ of post-recovery 1 ; and $20 \mathrm{~min}$ of post-recovery 2 . A $10 \mathrm{~min}$ rest period followed the collection of baseline data because of the effects of anticipatory anxiety in the period immediately preceding the anticipated threat. ${ }^{10-11}$ Research has shown that 20 min of HRV data correlates with 24-h RR interval variation using Holter monitoring, hence the use of 20min segments to note changes in SDNN. ${ }^{12}$ The STAI was completed by the participants at the beginning of each 20-min interval (i.e. baseline, stressor, recovery 1 and recovery 2). Figure 1 illustrates the testing protocol and measurements taken during these time intervals. Two different series of psychological stressors (stressor A and stressor B) were used during the pre-tests and post-tests. ${ }^{13}$ These tests were randomly counter balanced across the participants from pre-test to post-test. Stressor A consisted of: (a) timed Wais-R Block Designs (Psychological Corporation, San Antonio, Texas, USA); (b) timed mathematics problems; and (c) a 5-min oral presentation. Stressor B consisted of: (a) stimulus and response cards from the Wechsler Intelligence Test (Psychological Corporation; San Antonio, Texas, USA); (b) timed anagrams; and (c) a 5-min oral presentation. The topic of the oral presentation differed in stressors A and B. For each timed task, three trials were allowed for each participant to complete the task correctly: repeated trial time intervals were reduced by $50 \%$ from the previous trial using a stop watch to time the trials. A panel of three reviewers dressed in white laboratory coats observed the participants during the stressors while sitting at a table. ${ }^{11,14}$ Pre-tests and post-tests followed the same protocol.

\section{Intervention}

Tai chi chuan was taught by an experienced TC instructor twice a week for 8 weeks. Each class session was approximately $60 \mathrm{~min}$ in length. The class format was as follows: 10-min warm-up; 40-min TC, and 10-min cool-down. The TC group also practised breathing techniques three times per week for 8 weeks as demonstrated in class by the instructor. A journal was given to each subject to record their adherence to the prescribed TC practices. Class attendance and adherence to the breathing exercises outside of class were monitored by 
the instructor.

\section{Data analysis}

Analysis of the data for this study was carried out using SPSS software (version 21 ; IBM Corporation, Chicago, USA). Before using SPSS for the analysis, HR and SDNN were calculated using Kubios HRV Analysis Software (University of Eastern Finland, Helsinki, Finland). All data were manually cleaned for artefacts using standardised procedures as described in the CardioPro Infinity Manual (Thought Technology, Montreal, Canada). Means (M) and SDs were calculated for age, weight, height and BMI. An independent $t$-test was conducted to assess pre-post differences between groups in the descriptive data, state and trait anxiety, SDNN, and coping styles. The SDNN was compared between groups pre-test using the average of all four measures taken in the pre-test (baseline, stressor, recovery 1 and recovery 2) and only the baseline for both groups to ensure that there were no initial differences between groups.

Even though HR was not a variable in terms of the hypotheses of the study, HR was examined statistically to understand the effectiveness of the stressor. The same analysis was used for HR, SDNN and STAI, although the purposes were different for HR. To examine the effects of TC on SDNN and STAI, respectively, a mixed plot ANOVA was conducted [2 (group) $\times 2$ (test) $\times 4$ (trial) with repeated measures on the last two factors]. Although all three coping style scores (PFSOC-RF, PFSOC-RC, PFSOC-SP) were measured with the use of a single 18 - item questionnaire, each coping style reflects a distinct strategy for coping. ${ }^{8,15}$ Thus, each coping style score was subjected to a 2 (group) $\times 2$ (test) ANOVA with repeated measures on the last factor.

\section{Results}

Thirty-five subjects were assessed for eligibility; eight subjects were excluded for reasons outlined in Figure 2. A total of 27 male college students were randomised to the TC $(n=14)$ and control $(n=13)$ groups. Full analysis was carried out for $n=9$ in the TC group and $n=11$ in the control group. There were no differences in any of the dependent variables between groups at baseline (Table 1).

In this study, HR was analysed as a descriptive statistic only to show the effectiveness of the stressor. The statistical results for HR indicated that the main effect for trial $[F(1.9$, $32.3)=21.945, P=0.000, \eta p^{2}=0.549$, power=1.0] and the interaction of trial and group $[F(1.9$, $32.3)=3.474, P=0.044, \eta p^{2}=0.162$, power $\left.=0.598\right]$ were significant. Pairwise comparisons (adjusted for multiple comparisons) showed significant differences only between trials one and two $(P=0.000)$, trials one and three $(P=0.025)$, trials two and three $(P=0.000)$, and trials two and four $(P=0.000)$. Mean and SD for HR are presented in Table 2.

\section{Outcome measures}

Spielberger's State-Trait Anxiety Inventory The statistical results for STAI ${ }^{7}$ scores indicated that the main effect for test $\left[F(1,18)=17.875, P=0.001, \eta p^{2}=0.498\right.$, power $\left.=0.979\right]$, the main effect of trial $\left[F(1,18)=7.765, P=0.003, \eta p^{2}=0.301\right.$, power $\left.=0.890\right]$ and the interaction of test and group $\left[F(1,18)=7.730, P=0.012, \eta p^{2}=0.300\right.$, power $\left.=0.748\right]$ were all statistically significant. For test, the pre-test mean was higher than the post-test mean $(34.35 \pm 1.69$ vs. $27.51 \pm 1.54$, respectively). Pairwise comparisons (adjusted for multiple comparisons) showed significant differences only between trials one and two $(P=0.046)$, trials one and four $(P=0.007)$, trials two and three $(P=0.015)$, and trials three and four $(P=0.001)$. No other 
significant differences were found (see Table 3).

Standard Deviation of Normal to Normal interval For the effect of TC on SDNN, there was a significant main effect for test $\left[F(1,18)=6.06, P=0.024, \eta p^{2}=0.252\right.$, power $\left.=0.664\right]$, a significant main effect for trial $\left[F(3,54)=9.32, P=0.000, \eta p^{2}=0.341\right.$, power $\left.=0.995\right)$ and a significant interaction between trial and group $\left[F(3,54)=2.814, P=0.048, \eta p^{2}=0.135\right.$, power $=0.645)$. There was also a significant main effect between groups $[F(1,18)=6.353$, $P=0.021, \eta p^{2}=0.261$, power $\left.=0.664\right)$. For test, the pre-test mean was lower than the post-test mean (69.15 \pm 5.21 vs. $79.58 \pm 4.29$, respectively). Pairwise comparisons for trial (adjusted for multiple comparisons) showed significant differences between trials one and two $(P=0.003)$, trials two and three $(P=0.001)$, trials two and four $(P=0.0000)$, and trials three and two $(P=0.001)$. The mean between groups were $85.015 \pm 6.27$ for the TC group and $63.71 \pm 5.67$ for the control group, respectively. No other significant differences were found (see Table 3).

Coping styles The results for coping styles (PFSOC-RF, PFSOC-RC, PFSOC-SP) ${ }^{8}$ indicated a significant interaction for group and test for the PFSOC-SP coping style $\left[F(1,18)=9.007, P=0.008, \eta p^{2}=0.334\right.$, power $\left.=0.810\right)$. This interaction indicates a dramatic shift in the suppressive style of coping for both groups. While the suppressive style of coping increased for the control group, the suppressive style of coping decreased for the TC group. No other significant differences were found (see Table 3 ).

\section{Discussion}

The findings of this study will be discussed in the order in which the data were reported above. As one reads the discussion of the findings, an awareness of the limitations should be kept in mind. The most significant limitation was the small group size. In addition, the findings can only be generalised to male college students between 18 and 45 years of age. This could be viewed as a limitation since so many older adults practise TC and the implications of this study would be beneficial to this population.

Nonetheless, the strengths of the study are manifold. The TC group was led by an instructor who has over 25 years' experience practising TC and 10 years' experience teaching TC. To our knowledge, this is the first study to examine changes in state anxiety as well as changes in biological responses to an acute stressor and coping styles following TC in male college students aged 18 and 45 years. The time interval used to examine changes in HRV was 20 min as opposed to $5 \mathrm{~min}$, which is often reported in the literature. As the period of monitoring decreases, SDNN estimates shorter and shorter cycle lengths. ${ }^{5}$

\section{Heart rate during the stressor}

Even though HR was not a variable in the hypotheses of the study, the effectiveness of the stressor must be documented. Based on the analysis, the stressor was effective. There was a $10 \%$ increase in HR from baseline for the TC group and 4\% increase for the control group during the stressor pre-test. For the post-test, there was a 7\% increase in HR from baseline during the stressor for the TC group but the HR response for the control group was only $1 \%$. What we find interesting is examining the difference between groups for STAI and SDNN in light of their HR response to a stressor. Changes in HR did not parallel concomitant changes in STAI and SDNN as would be expected.

\section{Spielberger's State-Trait Anxiety Inventory}

Interestingly, the TC and control groups flipped in terms of their state anxiety response (STAI scores) to a stressor following the intervention. This suggests that the TC group had learned 
to manage their subjective reactions to a stressor following TC; this change in perception was not seen in the control group. There was a $23 \%$ increase (35.44-43.56) in STAI from baseline during recovery 1 in the TC group at pre-test, but a 4\% drop (27.56-26.33) in STAI from baseline during recovery 1 post-test. For the control group, the percentages were 7\% (32.7335.18 ) and $8 \%$ (29.73-32.18), respectively. The perception of stress changed in the TC group following the intervention but not in the control group (even though HR responses were similar).

\section{Heart-rate variability: Standard Deviation of Normal to Normal intervals}

Since decreased HRV is associated with exposure to emotional stressors, we expected to see a drop in SDNN during the stressor. ${ }^{16,17}$ This drop in HRV confirms the effectiveness of the stressor. It is thought by the authors that the magnitude of rebound in variability following the stressor is more important than the drop in variability during the acute stress. The TC group had increased the variability in their HR following TC even though there was a large drop in variability post-test during the stressor for the TC group. There was an impressive rebound in HRV post-test during recovery. Variability in HR represents a stable biological system with a complex pattern of overlapping oscillations that can respond to the demands placed on the system. ${ }^{16}$ When a biological variable rises, a regulatory reflex is triggered that makes it fall again. These oscillations represent the body's self-regulatory mechanisms to maintain homeostasis. On the other hand, decreased HRV is a marker of impaired autonomic function and impaired cardiovascular regulation. ${ }^{5}$

The significant interaction between trial and group suggests that the two groups changed differently across trials. The TC group's post-test data shows larger variation (i.e. larger amplitude of variation) than the control group. The oscillations in HRV were much more robust for the TC group following the intervention. The high variability in the TC group's HR during recovery post-test represents an adaptive response in the cardiovascular system, a beneficial effect of the TC intervention.

\section{Suppressive Problem-Focused Styles of Coping}

The results for coping styles revealed a striking change in terms of the suppressive style of coping (PFSOC-SP) for both groups. The change was inversely related; the scores for the suppressive style decreased significantly for the TC group while the suppression of problematic emotions increased for the control group. The suppressive style of coping is described as the tendency to deny the existence of problems as well as avoid coping activities; this is in contrast to the analytical reflective or emotionally focused reactive style. ${ }^{8}$ Of particular importance is the fact that, unfortunately, no previous study exists for comparison. This is an exciting result of this study, the findings of which need to be replicated in future studies.

\section{Conclusions}

In conclusion, our hypotheses were supported in this study. Implications of our study are that more people will use physical activity, such as TC, as a means of coping with stressors in their lives and to increase health and well-being. In Healthy People 2020, ${ }^{18}$ the United States Department of Health and Human Services stated that a primary objective is to increase the level of physical activity in adults. We recommend TC as an effective means to increase physical activity and promote health. Our reasons based on our study results are as follows: 
- $\quad$ Tai chi has a positive effect on biomarkers of stress, specifically HRV, as measured using the metric SDNN.

- $\quad$ Tai chi has an effect on subjective measures of stress as measured by STAI.

- $\quad$ Coping styles, as measured by the PFSOC questionnaire, shifted towards a healthier paradigm.

Conflict of interest The authors have no financial or personal relationships that may bias our work, nor do we have any conflicts of interest to declare.

\section{Acknowledgements}

We would like to acknowledge Dr Eneko Larumbe Zabala for his contributions to the figures and tables in the manuscript.

\section{References}

1 McEwen BS. Allostasis and allostatic load: implications for neuropsychopharmacology. Neuropsychopharmacol 2000; 22: 108-24.

2 McEwen BS, Stellar E. Stress and the individual: mechanisms leading to disease. Arch Intern Med 1993; 153: 2093-2101.

3 Sterling P, Eyer J. Allostasis: A new paradigm to explain arousal pathology. In: Fisher S. Reason JT. Handbook of Life Stress, Cognition, and Health. Chichester, UK: John Wiley \& Sons; 1988. pp. 629-649.

$4 \quad$ Lehrer P. Biofeedback training to increase heart rate variability. In: Lehrer P, Woolfolk RL, Sime WE (Eds). Principles and Practice of Stress Management. New York, New York: The Guilford Press, 2007. pp. 227-247.

5 Task Force of the European Society of Cardiology the North American Society of Pacing Electrophysiology. Heart rate variability: standards of measurement, physiological interpretation, and clinical use. Circulation 1996; 93: 1043-65.

6 Wang YT, Taylor L, Pearl M, Chang LS. Effects of tai chi exercise on physical and mental health of college students. Am J Chin Med 2004; 32: 453-9.

7 Spielberger CD. Manual for the State-Trait Anxiety Inventory. Palo Alto, CA: Consulting Psychologists Press, 1983.

8 Heppner PP, Cook SW, Wright DM, Johnson WC. Progress in resolving problems: A problem-focused style of coping. J Counseling Psychol 1995; 42: 279-93.

9 Shephard, R J. PAR-Q, Canadian home fitness test and exercise screening alternatives. Sports Med 1988; 5: 185-95.

10 Beatty MJ, Behnke RR. Effects of public speaking trait anxiety and intensity of speaking task on heart rate during performance. Hum Communication Res 1991; 18: 147-76.

11 Kirschbaum C, Pirke KM, Hellhammer DH. The Trier Social Stress Test - a tool for investigating psychobiological stress responses in a laboratory setting. Neuropsychobiol 1993; 28: 76-81.

12 Del Pozo JM, Gevirtz RM, Scher B, Guarneri E. Biofeedback treatment increases heart rate variability in patients with known coronary artery disease. Am Heart J 2004; 147: G1-G6.

13 Wechsler, D. WAIS-R. Manual. San Antonio, TX: Psychological Corporation, 1981.

14 McComb J, Norman R, Qian X et al. Neuroendocrine responses to psychological stress 
in eumenorrheic and oligomenorrheic women. Stress 2006; 9: 1-11.

15 Tacón AM, McComb J, Caldera Y, Randolph P. Mindfulness meditation, anxiety reduction, and heart disease: a pilot study. Fam Community Health 2003; 26: 25-33.

16 Giardino N, Lehrer PM, Feldman J. The role of oscillations in self regulation: their contribution to homeostasis. In: Kenney D, McGuigan FJ (Eds). Stress and Health: Research and Clinical Applications. New York: Hardwood Publishers, 2000. pp. 2752.

17 Lehrer PM, Woolfolk RL, Sime WE. Principles and Practice of Stress Management, (3rd edn). New York: Guilford Press, 2007.

18 U.S Department of Health and Human Services. Healthy People 2010: Understanding and Improving Health (2nd edn). Washington, DC: U.S. Government Printing Office, 2000.

Jacalyn J Robert-McComb, BS, MAT, PhD, FACSM, Certified ACSM ETT, CEP, \& PD, Professor, Department of Health, Exercise \& Sport Sciences, Texas Tech University, Box 43011, Lubbock, TX 79409-3011, USA.

E-mail: jacalyn.mccomb@ttu.edu

Ming Chyu, BS, MS, PhD, Professor, Department of Mechanical Engineering, Texas Tech University, Box 41021, Lubbock, TX 79409-1021 USA.

E-mail: m.chyu@ttu.edu

Anna Tacón, BS, MS, PhD, Associate Professor, Department of Health, Exercise \& Sport Sciences, Texas Tech University, Box 43011, Lubbock TX 79409-3011, USA.

E-mail: anna.tacon@ttu.edu

Reid Norman, PhD, Professor of Pharmacology, Texas Tech University Health Science Center, $36014^{\text {th }}$ St. Stop 6592 Lubbock, TX 79430-6592, USA.

E-mail: reid.norman@ttuhsc.edu

Figure Legends:

Figure 1 Protocol for the pre-test and post-test stressor.

Figure 2 Flowchart showing selection and exclusion process, as recommended by CONSORT. 
Table 1 Descriptive statistics and baseline data for the control $(n=11)$ and tai chi $(n=9)$ groups

\begin{tabular}{lccc}
\hline \multicolumn{4}{c}{ Pre-test differences between the control and tai chi groups } \\
\hline \multicolumn{1}{c}{ Variable } & Control & Tai chi & $\begin{array}{c}\text { Significance } \\
\text { (two-tailed) }\end{array}$ \\
Age & $21.7 \pm 1.0$ & $23.0 \pm 5.1$ & 0.429 \\
Height (m) & $1.8 \pm 0.1$ & $1.8 \pm 0.1$ & 0.398 \\
Weight (kg) & $85.5 \pm 15.7$ & $86.7 \pm 17.9$ & 0.876 \\
BMI (kg/m2) & $26.4 \pm 3.5$ & $27.8 \pm 6.4$ & 0.53 \\
Trait anxiety & $31.5 \pm 6.1$ & $37.8 \pm 9.2$ & 0.86 \\
State anxiety & $31.6 \pm 6.1$ & $37.8 \pm 9.2$ & 0.086 \\
Baseline SDNN & $55.89 \pm 20.75$ & $78.51 \pm 30.66$ & 0.065 \\
Average SDNN & $59.4 \pm 19.3$ & $78.9 \pm 27.2$ & 0.077 \\
Reflective PFSOC & $21.67 \pm 2.69$ & $24.36 \pm 2.69$ & 0.129 \\
Suppressive PFSOC & $13.78 \pm 3.83$ & $10.73 \pm 3.83$ & 0.563 \\
Reactive PFSOC & $12.89 \pm 4.68$ & $11.36 \pm 2.91$ & 0.091 \\
\hline
\end{tabular}

PFSOC, Problem-Focused Styles of Coping; SDNN, Standard Deviation of Normal to Normal intervals. 
Table 2 Means and SDs for heart rate for the control $(n=11)$ and tai chi $(n=9)$ groups

\begin{tabular}{ccccccccc}
\hline \multicolumn{3}{c}{ Pre-test } & \multicolumn{3}{c}{ Post-test } \\
\hline & Pre-baseline & Pre-stressor & Pre-recovery 1 & Pre-recovery 2 & Post-baseline & Post-stressor & Post-recovery 1 & Post-recovery 2 \\
Control & $74.19 \pm 9.79$ & $77.81 \pm 7.46$ & $72.76 \pm 9.36$ & $72.83 \pm 8.16$ & $71.83 \pm 9.47$ & $72.89 \pm 8.69$ & $70.69 \pm 7.90$ & $71.86 \pm 9.17$ \\
Tai chi & $71.59 \pm 10.34$ & $78.41 \pm 15.04$ & $69.93 \pm 10.72$ & $70.32 \pm 10.09$ & $68.53 \pm 7.59$ & $74.23 \pm 9.05$ & $67.36 \pm 7.54$ & $67.69 \pm 6.76$ \\
\hline
\end{tabular}


Table 3 Means and SDs for State-Trait Anxiety Inventory, Standard Deviation of Normal to Normal interval and Suppressive Problem-Focused Styles of Coping across the study for the control $(n=11)$ and tai chi $(n=9)$ groups

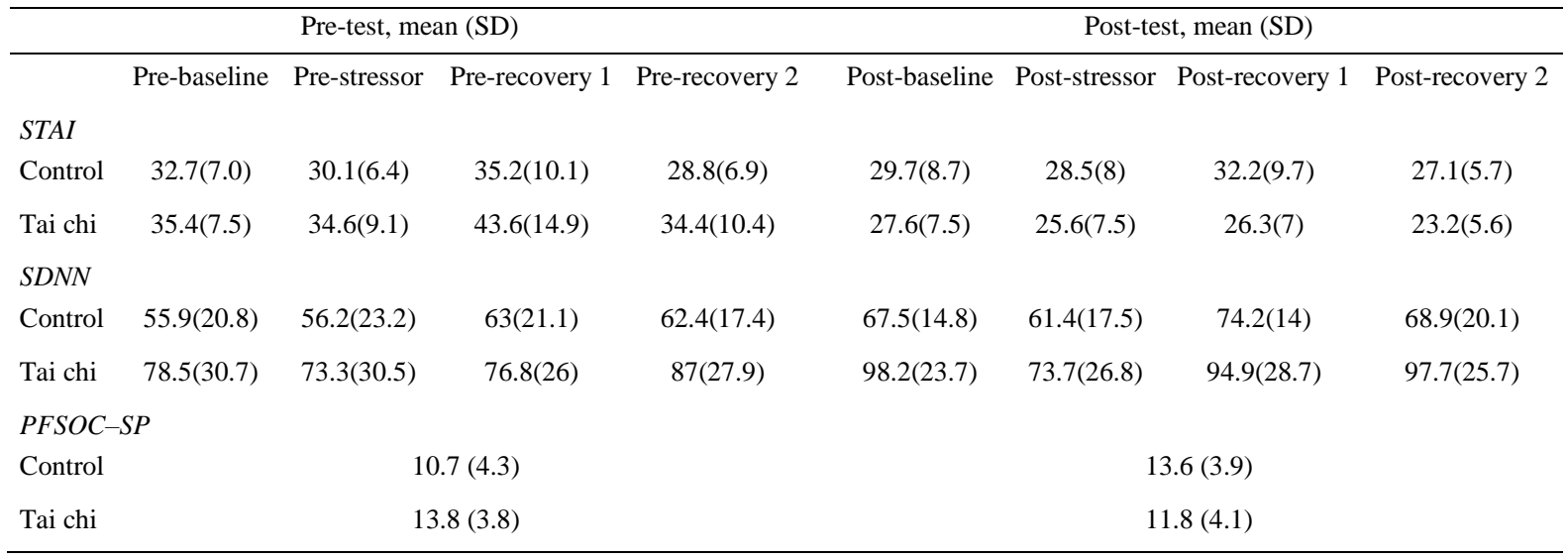

PFSOC-SP, Suppressive Problem-Focused Styles of Coping; SDNN, Standard Deviation of Normal to Normal interval; STAI, Spielberger's State-Trait Anxiety Inventory. 